\title{
Automated Influence Maintenance in Social Networks: an Agent-based Approach
}

\author{
Weihua Li, Quan Bai, Minjie Zhang and Tung Doan Nguyen
}

\begin{abstract}
Social influence modelling and maximization appear significant in various domains, such as e-business, marketing, and social computing. Most existing studies focus on how to maximize positive social impact to promote product adoptions based on static network snapshots. Such approaches can only increase influence in a social network in short-term, but cannot generate sustainable or long-term effects. In this research work, we study on how to maintain long-term influence in a social network and propose an agent-based influence maintenance model, which can select influential nodes based on the current status in dynamic social networks in multiple times. Within the context of our investigation, the experimental results indicate that multiple-time seed selection is capable of achieving more constant impact than that of one-shot selection. We claim that influence maintenance is crucial for supporting, enhancing and assisting long-term goals in business development. The proposed approach can automatically maintain long-lasting impact and achieve influence maintenance.
\end{abstract}

Index Terms-Influence maintenance, influence diffusion, long-lasting influence, agent-based modelling

\section{INTRODUCTION}

W ITH the prevalence and advancement of the Internet, on-line social networks have become an important and efficient channel for information propagation. The propagation relies on one of the social phenomena, i.e., social influence, indicating that one's opinions or behaviours are affected by his or her contactable neighbours in the social network [1], [2]. Influence message is a common and concrete representation of social influence, which 'travels' rapidly through the network topologies via users' sharing and posting behaviours. By leveraging the power of social influence, a great many business owners attempt to expand the market and increase the brand awareness through the 'word-of-mouth' effect (or called viral marketing) [3]. In recent years, influence maximization draws tremendous attention to both researchers and domain experts. Influence maximization attempts to identify a set of influential users committed to spreading a piece of influence message to their neighbours, such as adopting a product, expecting that they can propagate influence and maximize the positive impact across the entire network [4]. The selected group of influencers is called seed set, and the seeding process is named as seed selection.

From a business perspective, influence maximization corresponds to short-term marketing effects, which tend to cause sudden profit spikes that rarely last [5]. Whereas, long-term marketing is typically more beneficial since it emphasizes on long-term and sustainable business goals.

- W. Li, Q. Bai and T.D. Nguyen are with the School of Engineering, Computer Mathematical Sciences, Auckland University of Technology, New Zealand E-mail: weihua.li@aut.ac.nz; quan.bai@aut.ac.nz; tung.nguyen@aut.ac.nz;

- M. Zhang is with the School of Computing and Information Technology, University of Wollongong, Australia

E-mail:minjie@uow.edu.au
Specifically, long-term influence can establish brand awareness and continually produce results even years down the road; thus, without having long-term marketing strategies, short-term success may be short-lived [6]. Motivated by this background, in this research, we aim to achieve constant impact for long-term marketing by investigating the preservation of a particular type of influential situation or status, called influence maintenance.

There are many limitations for short-term (or even oneshot) influence maximization when being utilized in real business cases. First, it focuses on how to maximize the influence of one-shot investment. Based on the risk management theory and best practice [7], with the same budget, the multiple-time investment could enable a better business strategy. In this way, the next action can be planned and carried out based on the outcome of the previous investment. For example, in a stock market, very few investors purchase stocks with all the money at only one time. Second, a great many business owners intend to expand the lifespan of influence, so that the brand awareness can be enhanced and increased in the long run [8]. Influence maintenance not only cares about the quantity of users being affected but also considers constant influence impact.

Influence maintenance needs to be supported by a formal influence diffusion model which possesses two attributes: (1) the model is capable of capturing the temporal feature of a social network; (2) the model can monitor the status of a particular influence. On the other side, in most existing on-line social media applications, information cannot be delivered to the users directly, but cached in individual's message repository, pending for users to access. The timeliness of a particular influence message becomes an important factor to be considered. More specifically, an individual reading list in on-line social networks, such as Weibo $^{1}$, is typically presented as a stack, which turns out

1. http://www.weibo.com 
to be last-post-first-read. Thus, the accessing priority of a particular message keeps decreasing over time, and posting or sharing behaviours are not supposed to be triggered without reading it.

In [9], we conducted very preliminary research work on modelling maintaining influence under a particular social context. In this paper, we systematically elaborate and formulate the influence maintenance problem, which tends to maximize the constant impact of a particular influence by considering time-series. Meanwhile, a decentralized influence propagation model, i.e., the Agent-based Timeliness Influence Diffusion (ATID) model, is proposed. In the ATID, the diffusion process is considered as a networked evolutionary phenomenon, users are modelled as autonomous agents, and each maintains its local information incorporating friendship affiliation list, message repository and posting histories. Furthermore, we propose the Timeliness Increase Heuristic (TIH) algorithm for solving the influence maintenance problem. Extensive experiments are conducted by using three real datasets. The experimental results show that: (1) multiple-time selection can maintain influence better than one-shot selection; and (2) the TIH algorithm outperforms the other traditional seed selection algorithms regarding maintaining influence in social networks; and (3) seed-set variation is associated with both selection approaches and network properties. To summarize, the contributions of this research work are as follows.

- We formally defined the influence maintenance problem. To the best of our knowledge, this is the first literature describing the maintenance of influence in on-line social networks, which is significantly different from the adaptive influence maximization problem (clarified in the related work).

- We proposed a novel decentralized influence diffusion model to accommodate to the influence maintenance problem. The proposed model is capable of capturing two major elements for maintaining longlasting influence, i.e., the temporal feature of a social network and the status of a particular influence.

- We proposed a novel timeliness-based seed selection algorithm to maximize the influence lifespan.

The rest of this paper is organized as follows. Section 2 reviews the literature related to this research work. Section 3 introduces the preliminaries, formal definitions and problem description. Section 4 systematically elaborates the influence maintenance using the proposed decentralized diffusion model, and the TIH algorithm is also described. In Section 5, experimental results are presented to evaluate the performance of the proposed model. Our conclusions and future works are detailed in Section 6.

\section{Related Work}

\subsection{Adaptive Influence Maximization}

A rich body of research works has been devoted to the influence maximization problem over the past ten years [4], [10]. The majority of these studies fall into either fullfeedback or non-feedback models [11]. In the former, all the seeds are committed based on the networked features or specific heuristics. Namely, there is no adaptive seed selection policy applied. Whereas, the latter utilizes the observations during the seeding process, where the rules for identifying influencers are also known as adaptive policies. Based on the full-feedback model, some researchers extend the influence maximization problem by exploring the adaptive budget allocations [11], [12], [13]. Hatano et al. address budget allocation for maximizing influence by considering adaptive strategies [14]. Yang et al. model the continuous influence maximization problem and devise a coordinate descent framework [15]. Similarly, Rodriguez and Schölkopf study influence maximization in continuous time diffusion networks by developing INFLUMAX model that accounts for the temporal dynamics underlying diffusion process [16].

Our research work departs from the body of the studies mentioned above mainly in two aspects. First, the existing studies focus on investigating adaptive policies on the basis of the concept of adaptive submodularity [11]. Whereas, we concentrate on modelling the influence maintenance, achieving a constant impact by considering the timeliness degrees, though adaptive seeding algorithms are proposed to accommodate to the model. Second, these research works do not give a clear concept of time-series, and the networked evolutionary trend driven by influences is not captured. While, in this paper, the time-series can be presented and the global observation of a social network status can be captured since Agent-Based Modelling (ABM) [17], [18] has been applied in our model.

\subsection{Dynamic Social Streams}

Dynamics is one of the major features of social networks. Users join and quit, and links are forming and vanishing over time. The influence propagation tendencies among the users also can be altered dramatically with the involvement of any breaking news. Many research works have been dedicated to the dynamic social streams, which aim to investigate the possible solutions for real-time influence maximization in a dynamic environment. Konstantin et al. present STRIP, the first streaming method computing influence probabilities [19]. Subbian et al. propose an influencequery framework to mine influencers in a time-sensitive fashion from streaming social data [20]. Wang et al. propose the Influential Checkpoints framework and a Sparse Influence Checkpoints framework to tackle the stream influence maximization querying processing [21].

Whereas, nearly all the literature of dynamic social streams still focus on the mining the influencers and enlarging the global activation coverage, but fail to track the status of any influence message. By contrast, the objective of influence maintenance is set to maintain the popularity of a particular influence message in a dynamic environment.

\subsection{Influence Diffusion Modelling}

Most researchers investigate influence diffusion and influence maximization problems based on two popularly adopted influence diffusion models, i.e., Independent Cascade (IC) model and Linear Threshold (LT) model [4]. Many studies are conducted under various extended influence diffusion models. Wang et al. propose the IMIC-OC model to explain how users build opinions during the process of 
information spreading [22]. Goyal et al. research learning influence probabilities in social networks based on the users' past actions, and successfully predict the time by which a user may be expected to perform an action [23]. Tang et al. propose topical affinity propagation to model the topic-level social influence and measure the strength quantitatively [24]. Chen et al. formulate the influence maximization problem by focusing on the temporal factors based on the heat diffusion model [25], a realistic model that simulates the social influence in accordance with a physical phenomenon, i.e., heat flow [26].

Most of the existing research works oversimplify the influence diffusion process, and the propagation models concentrate on the activation state of each individual. Whereas, the users' features and behaviours affecting the influence acceptance have not been considered. Moreover, the dynamic status of influence messages over time is neglected. By contrast, the proposed ATID model for influence maintenance is decentralized, focusing on modelling individuals' personalized traits and behaviours. Furthermore, the ATID is capable of capturing the evolutionary network trend based on timeseries, as well as the status of influence messages.

\subsection{Agent-based Modelling for Influence Diffusion}

Agent-Based Modelling (ABM) has demonstrated many advantages in modelling complex systems, simulating continuous variations and analysing the trend of a particular phenomenon [17], [18]. Moreover, it is more suitable for exploring the macro world through defining a micro level of a social system [27], [28]. Some researchers model the influence diffusion in a social network by leveraging ABM. Jiang et al. survey the influence diffusion in social networks from a multi-agent perspective [29]. Li and Tang analyse the group polarization based on ABM [30]. Van Maanen and Van der Vecht propose a multi-disciplinary approach for studying on-line social network influence [31]. Similarly, Li et al. propose an agent-based influence diffusion model, where the influence propagation demonstrates an evolutionary process, and the model is applicable in a dynamic environment and functions even without the network topology [32]. In their studies, the factors affecting the activation cost are considered, including individual's personalities, i.e., the degree of stubbornness, predisposition, i.e., prior commitment level, and social pressure. Li et al. exploit influence maximization using a novel decentralized approach, i.e., the stigmergy-based influence maximization model, where the influence propagation process is modelled as ants crawling across the network topology [33]. However, the message timeliness feature has been ignored in nearly all of these studies. With an exception, Han et al. propose a novel algorithm for addressing the influence maximization problem, which incorporates time delay for timeliness, opportunistic selection for acceptance ratio and broad diffusion for influence breadth [34]. Nevertheless, their proposed BICOT model in [34] neglects the long-term trend of a social network.

On the other side, most agent-based models for influence diffusion are user-centred, which follow the rule that a user activates users through direct interactions. The evident disadvantages are reflected in two aspects, i.e., lacking the model of influence instances and having difficult in estimating the influence probabilities. The former fails to track the state of influence; the latter shows a non-trivial task in calculating the weight of influential links, i.e., the 'cost' of activating one user by the neighbours [23], [35].

Different from the studies and models discussed above, the proposed ATID model captures the properties of the influences existed in the same environment as that of the individuals'. Therefore, the observations of the disseminated influence messages can also be reflected from the ATID model. Moreover, the influence activation is channelled through accessing messages in the repository, which mitigates the complexity of modelling the influential relationships among the individuals.

\section{Preliminaries and Problem Formulation}

\subsection{Social Networks and Newsfeed}

Most on-line social networks can be classified into two categories, depending on whether the newsfeed is re-organized.

First, some popular on-line social networks, such as Facebook ${ }^{2}$, create personalized activity feeds for increased interactions and content contributions [36]. For example, Facebook previously employed the EdgeRank algorithm ${ }^{3}$ to determine which stories appear as newsfeed for each user by considering three original elements, i.e., affinity, weight and time decay [37]. Therefore, to maximize the impact of a particular influence, social media marketers need to stay informed of the changes to the latest newsfeed algorithms. Nowadays, newsfeed algorithms have become much more sophisticated. For example, Facebook has begun to employ a more complex ranking algorithm based on machine learning [36], [37], [38]. In this sense, it is nearly impossible for researchers to investigate the influence diffusion modelling in such social networks, as the outcome is much dependent on the newsfeed algorithms.

Second, on-line social networks, like $\mathrm{WeChat}^{4}$, enable users to share daily moments with friends. The newsfeed is generated instantly based on the timeline. Moreover, the social interactions among the individuals, such as 'comments' and 'like', are only visible if friendship connections are established. Different from Facebook, such kind of social networks allow duplicate messages propagating through the network, and no newsfeed algorithms are applied. Moreover, 'posting a message' or 'forwarding a message' can be regarded an influential behaviour, while 'like' and 'comments' weigh less due to the visibility and privacy restrictions.

In this research, we mainly focus on the second category of social networks and investigate influence maintenance, where the timeliness degree of a message plays a pivotal role in organizing the newsfeed.

\subsection{Agent-based Influence Diffusion}

ABM simulates the influence diffusion process by emphasizing individualized features and behaviours. Users in social networks have been modelled as autonomous interactive

\footnotetext{
2. https://www.facebook.com/

3. http:/ / edgerank.net/

4. http://www.wechat.com/en/
} 


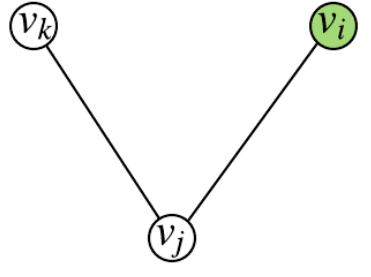

(a) Social Network Graph

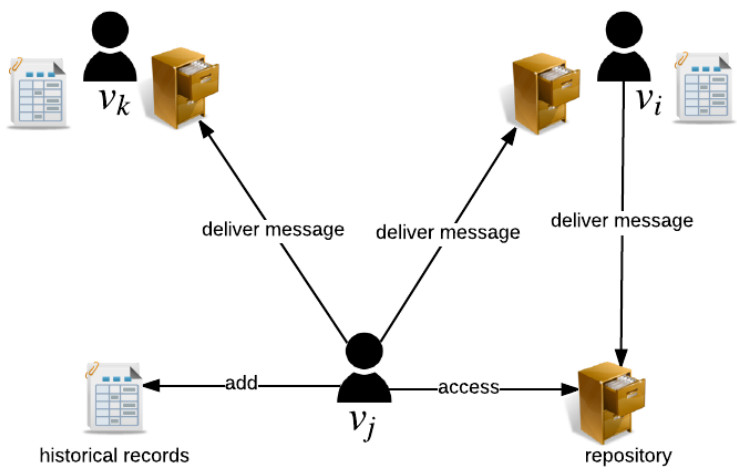

(b) Agent-based Influene Diffusion Model

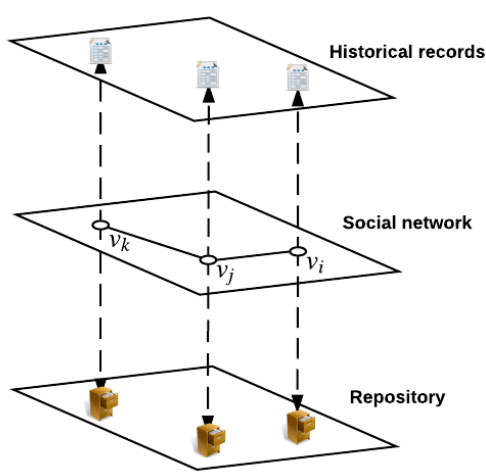

(c) Influence Diffusion Facets

Fig. 1. General Idea of the Proposed Model

agents, and they have their interests and behaviours. Based on the influence theory, homophily and influence are driven by the users' preferences. Thus, individuals have different tendencies of reading and posting different types of topics [39]. The messages wrapped with influence are supposed to be delivered to the repository of corresponding recipients, where the repository is filled with the influence messages from the neighbours. Each agent has a different frequency of accessing its repository. Based on agent's preference and message timeliness degree (see Definition 4), the agent determines whether the information is to be shared with its adjacent neighbours. If an agent is influenced (activated), i.e., posting action is triggered, then, the influence message reaches its neighbours' repositories. Whereas, in the recipient's repository, the timeliness degree of this message keeps decreasing over time, but this will be refreshed if the repository owner is activated or the same message has been received again.

In Figure 1, a toy example has been demonstrated, which represents the general idea of our proposed model. Figure 1a shows an ordinary social network graph in traditional influence diffusion models. Let $v_{i}$ be an initial influencer who attempts to activate $v_{j}$ with a certain success rate. While $v_{j}$ intends to influence its adjacent neighbour $v_{k}$ if $v_{j}$ is activated by $v_{i}$. Figure $1 \mathrm{~b}$ describes our model from a microscopic point of view. Individual's influence activation is achieved by accessing the repository. More specifically, if a user is influenced (activated), the influence message is supposed to be delivered to all the neighbours' repositories. Meanwhile, this message is archived as one of the sender's historical records. From a macroscopic viewpoint, apart from the topological structure of a social network, two more factors are affecting the influence propagation, i.e., the historical records and the repository, which is illustrated in Figure 1c.

Since the individual's activation is achieved through reading influence messages in the repository, the user-user influential relationship in this model does not directly affect the influence acceptance. Namely, the model is developed based on the assumption that the influence activation is only driven by the existing messages in the repository and past posting behaviours. Moreover, with consideration of
TABLE 1

Frequently Used Notations

\begin{tabular}{ll}
\hline Notation & Description \\
\hline$v_{i}$ & user agent \\
$m s g_{p}$ & influence message \\
$\varphi$ & timeliness degree \\
$r$ & influence attenuation constant \\
$\lambda$ & the speed of influence decay \\
$R_{v_{i}}$ & incoming message repository of $v_{i}$ \\
$H_{v_{i}}$ & historical records of $v_{i}$ \\
$A$ & seed set \\
$\varrho_{m s g_{p}}$ & global activation coverage (GAC) of $m s g_{p}$ \\
$\xi_{m s g_{p}}^{t_{n}}$ & global timeliness degree (GTD) of $m s g_{p}$ at $t_{n}$ \\
$\Omega_{m s g_{p}}$ & global cumulative timeliness degree (GCTD) of $m s g_{p}$ \\
$\Delta \Omega$ & incremental timeliness contribution \\
$P()$. & probability \\
$g()$. & timeliness gain \\
\hline
\end{tabular}

different activation cost for each agent, the model would become a rather complicated one.

The detailed modelling will be elaborated in Section 4 . Before moving on to the technical parts of this paper, we summarise the frequently used notations in Table 1.

\subsection{Formal Definitions}

Definition 1: A user agent $v_{i},\left(v_{i} \in V\right)$ is defined as a vertex in a social network $G=(V, E)$, where $V=\left\{v_{1}, \ldots, v_{n}\right\}$ denotes a set of agents and $E$ represents a set of edges, $E=\left\{e_{i j} \mid 1 \leq i, j \leq n\right\}, i, j \in \mathbb{N}^{+},\left\{v_{i}, v_{j}\right\} \subseteq V . v_{i}$ has a neighbour set $\Gamma\left(v_{i}\right)$. If agent $v_{j}$ is a neighbour of $v_{i}$, then $\left\{e_{i j}\right\} \subseteq E, v_{j} \in \Gamma\left(v_{i}\right)$. While, $E_{v_{i}}$ indicates the edge set connected with $v_{i}$, where $E_{v_{i}}=\left\{e_{i j} \mid v_{i} \neq v_{j} \wedge v_{j} \in \Gamma\left(v_{i}\right)\right\}$. $|V|$ and $|E|$ denote the cardinality of agents and edges respectively. The affiliation information is maintained by each agent locally. In addition, agent $v_{i}$ has a binary state $s_{v_{i}}^{m s g_{p}}$ towards a particular influence message $m s g_{p}$ (see Definition 3), where $s_{v_{i}}^{m s g_{p}} \in\{0,1\}$, representing inactive and active, respectively.

Definition 2: Environment $\varepsilon_{v_{i}}$ is an ego network representing the local influence diffusion context or the local view of a particular agent $v_{i}$. The environment of $v_{i}$ is denoted by using a four-tuple, $\varepsilon_{v_{i}}=\left(\Gamma\left(v_{i}\right), E_{v_{i}}, R_{v_{i}}, H_{v_{i}}\right)$, where $R_{v_{i}}$ and $H_{v_{i}}$ represent the repository and historical 
records of $v_{i}$, respectively (see Definitions 4 and 5). Each agent is capable of accessing all the resources in its environment.

Definition 3: Influence message $m s g_{p}$ is defined as a particular piece of information sent from one person to his or her contactable recipients, affecting their opinions or behaviours. It is a common and concrete representation of social influence in on-line social networks. In the current settings, each influence message $m s g_{p}$ belongs to a particular topic $\tau_{x}$, i.e., $m s g_{p} \in \tau_{x}$. If agent $v_{i}$ is influenced after accessing $m s g_{p}$, then $s_{v_{i}}^{m s g_{p}}:=1$; meanwhile, $v_{i}$ attempts to deliver the influence message to the repositories of neighbours $\Gamma\left(v_{i}\right)$.

Definition 4: Timeliness degree of an influence message is a real value, describing the position of an influence message in a user's repository at a particular time. Timeliness degree not only reflects the status of the influence message, but also implies whether a specific piece of news arrives at a suitable time. In reality, it happens more than often that users check the friend-circle or moments update right after a message has been posted. Subsequently, this influence message has a higher chance to draw the user's attention than that of the others. Mathematically, we define the timeliness degree of message $m s g_{p}$ in $v_{i}$ 's repository at time $t_{m}$ using the notation $\varphi\left(v_{i}, m s g_{p}, t_{m}\right)$.

Inspired by the behaviour analysis approach introduced in [40], [41], we assume that the effect of influence satisfies the principle of natural decay; thus, the exponential decay, i.e., $e^{-r}$, can be leveraged to describe the attenuation of influence, where $r$ denotes the attenuation constant. Suppose message $m s g_{p}$ has been delivered to $v_{i}$ 's repository at $t_{b}$, then the timeliness degree is formulated in Equation 1.

$$
\varphi\left(v_{i}, m s g_{p}, t_{m}\right)=e^{-r \cdot(m-b)}
$$

The timeliness degree of any message equals to 1 when arriving at the repository, i.e., $m=b$, and starts to decrease over time. Therefore, the speed of influence decay $\lambda$ is described in Equation 2, which shows the speed is gradually slowing down.

$$
\begin{aligned}
\lambda & =\varphi\left(v_{i}, m s g_{p}, t_{m-1}\right)-\varphi\left(v_{i}, m s g_{p}, t_{m}\right) \\
& =e^{-r \cdot(m-1-b)}-e^{-r \cdot(m-b)} \\
& =\left(e^{r}-1\right) \cdot e^{-r \cdot(m-b)}, m \geq b
\end{aligned}
$$

We assume $m s g_{p}$ is supposed to be ignored by agent $v_{i}$ after time $t_{e}$, subject to $e \in \mathbb{N}, e>m-b$ and $\varphi\left(v_{i}, m s g_{p}, t_{e}\right) \geq \sigma\left(m s g_{p}\right)$, where $\sigma\left(m s g_{p}\right)$ denotes the valid timeliness degree threshold of $m s g_{p}$. Likewise, the higher timeliness degree, the greater probability that the influence message can be accessed by the user when visiting the repository.

Definition 5: Repository $R_{v_{i}}^{t_{m}}=<r_{1}, r_{2}, \ldots, r_{n}>$ refers to a cached container of agent $v_{i}$ at time step $t_{m}$. It incorporates all the valid incoming messages from neighbours $\Gamma\left(v_{i}\right)$ to agent $v_{i}$. Each agent has a different frequency of accessing the repository. An element in $R_{v_{i}}^{t_{m}}$ can be represented as a three-tuple, i.e., $r_{k}=\left(v_{j}, m s g_{p}, \varphi\right)$, where $v_{j}$ denotes the agent who posts the influence message $m s g_{p}$, $v_{j} \in \Gamma\left(v_{i}\right) \cup\left\{v_{i}\right\}$ and $\varphi \geq \sigma\left(m s g_{p}\right)$. For simplification purposes, we regard $\varphi$ as the timeliness degree of the corresponding message at $t_{m}$, which is equivalent to $\varphi\left(v_{i}, m s g_{p}, t_{m}\right)$.

Definition 6: Historical records refer to past outgoing influence messages delivered from a particular user to the neighbours. Historical records $H_{v_{i}}=\left\{t x n_{1}, t x n_{2}, \ldots, t x n_{n}\right\}$ is defined as a collection of user $v_{i}$ 's past sharing transactions, i.e., posted messages. An element of $H_{v_{i}}$ can be denoted by a three-tuple, i.e., $t x n_{n}=\left(m s g_{p}, \varphi, t_{m}\right)$, where $\varphi$ represents the message timeliness degree when posted (clarified in Definition 5), $\varphi \geq \sigma\left(m s g_{p}\right)$. While, $t_{n o w}$ refers to the current time step, and $\Delta t$ describes the valid lifespan of a transaction, $t_{\text {now }}-t_{m} \leq \Delta t$. Given $t_{\text {now }}-t_{m}>\Delta t$, the corresponding transaction is supposed to be removed from the collection. Historical records $H_{v_{i}}$ is also an implication of agent $v_{i}{ }^{\prime}$ s interests or preferences.

\subsection{Problem Description}

Influence maintenance in this paper is defined as the process of preserving a particular type of influential situation or the status of influence being preserved. The concept is derived from influence maximization. Specifically, given a finite budget $k$ (seed set size) and a limited time span $\left[t_{0}, t_{m}\right]$, an investment (seed selection) occurs once every $n$ time steps, thus, the investment time steps $I=\left\{t_{N \times n} \mid N \in \mathbb{N} \wedge N \times n<\right.$ $m\}$, where $t_{N \times n}$ represents a particular seed selection point. There are $|I|$ times of investment considered for maintaining the influence.

Influence maintenance aims to find a solution of identifying the seed set $A_{t_{N \times n}}$ for each time step $t_{N \times n}$ to maximize the influence lifespan of $m s g_{p}$. Thus, the selected seed set $A$ is a collection of seeds identified from each investment time step, i.e., $A=\left\{A_{t} \mid t \in I\right\}$ and

$$
\sum_{t \in t_{N \times n}}\left|A_{t}\right|=k
$$

We assume that the same amount of seeds are supposed to be selected for each selection point, and any seeds cannot be selected more than once. In other words, given $\left\{A_{i}, A_{j}\right\} \subseteq A,\left|A_{i}\right|=\left|A_{j}\right|, A_{i} \cap A_{j}=\emptyset$.

The Global Timeliness Degree (GTD) of $m s g_{p}$ at a particular time step $t_{n}$ is represented as $\xi_{m s g_{p}}^{t_{n}}$, which can be calculated by using Equation 4 . The popularity trend of a particular influence message can be reflected by connecting the GTD of the corresponding influence in each time step.

$$
\xi_{m s g_{p}}^{t_{n}}=\sum_{v_{i} \in V} \varphi\left(v_{i}, m s g_{p}, t_{n}\right)
$$

The overall effective influence lifespan of $m s g_{p}$ in the entire social network is evaluated by using Global Cumulative Timeliness Degree (GCTD) of a specific time span $\left[t_{0}, t_{m}\right]$, i.e., $\Omega_{m s g_{p}}$, which can be derived by using Equation 5 .

$$
\Omega_{m s g_{p}}=\sum_{t_{0}}^{t_{m}} \xi_{m s g_{p}}^{t_{n}}=\sum_{t_{0}}^{t_{m}} \sum_{v_{i} \in V} \varphi\left(v_{i}, m s g_{p}, t\right)
$$


The objective of influence maintenance is to maximize $\Omega_{m s g_{p}}$. Furthermore, the traditional influence effectiveness evaluation metrics, i.e., Global Activation Coverage (GAC), is taken into consideration as well. GAC of influence message $m s g_{p}$ is denoted using the notation $\varrho_{m s g_{p}}$, indicating the number of users in the social network getting affected or activated by $m s g_{p}$. It is formulated in Equation 6 .

$$
\varrho_{m s g_{p}}=\sum_{v_{i} \in V}\left|\left\{v_{i} \mid s_{v_{i}}^{m s g_{p}}=1\right\}\right|
$$

\section{Influence Maintenance Model}

\subsection{The Agent-based Timeliness Influence Diffusion (ATID) Model}

The ATID model is a decentralized influence diffusion model which utilizes the advantages offered by ABM. The influence propagation in social networks demonstrates a networked evolutionary pattern driven by individuals' actions. In this model, each agent maintains its ego-network and makes decisions of performing social activities based on both timeliness degree of the influence message and its preference.

There are many reasons to make a user to carry out a social behaviour, such as influence from neighbours in the same social networks, affected by any external events, or the user actively posts some messages without getting influenced by anybody [23]. In the proposed model, we assume users deliberately post messages after influenced by the neighbours, and each individual's repository and historical records contain enough evidence for statistical analysis. Furthermore, each user agent (e.g., $v_{i}$ ) has a different frequency of accessing its repository, i.e., $\operatorname{freq}\left(v_{i}\right)$, which can be calculated by using Equation 7. It can be seen that $\operatorname{freq}\left(v_{i}\right)$ is equivalent to the probability of $v_{i}$ accessing a particular message $m s g_{p}$ in its repository at time $t_{m}$, i.e., $P_{f}\left(v_{i}, m s g_{p}, t_{m}\right)$.

$$
\begin{aligned}
\operatorname{freq}\left(v_{i}\right)= & P_{f}\left(v_{i}, \operatorname{msg}_{p}, t_{m}\right), \\
& \text { subject to } \varphi\left(v_{i}, m_{s} g_{p}, t_{m}\right) \geq \sigma\left(m s g_{p}\right)
\end{aligned}
$$

One important task of influence diffusion modelling is to identify the probability of getting activated after reading message $m s g_{p}$ of topic $\tau_{x}$ at time $t_{m}$, where the influence probability may not remain constant independently of time [23]. Therefore, in the proposed model, a user agent has the capability of adapting its probability of posting message $m s g_{p}$ based on two major factors, i.e., the attention degree of influence message $m s g_{p}$ and the user preference derived from the latest $k$ posts. Therefore, the probability of user agent $v_{i}$ posting message $m s g_{p}$ at time $t_{m}$ can be estimated in Equation 8:

$$
P\left(m s g_{p} \mid R_{v_{i}}^{t_{m}}, H_{v_{i}}\right)=P\left(m s g_{p} \mid R_{v_{i}}^{t_{m}}\right) P\left(\tau_{x} \mid H_{v_{i}}, m s g_{p} \in \tau_{x}\right)
$$

In Equation 8, $P\left(m s g_{p} \mid R_{v_{i}}^{t_{m}}\right)$ represents the attention degree of influence message $m s g_{p}$ in $v_{i}$ 's repository at time $t_{m}$, i.e., the probability of getting attracted by $m s g_{p}$, which is associated with the message timeliness degree $\varphi\left(v_{i}, m s g_{p}, t_{m}\right)$. While $P\left(\tau_{x} \mid H_{v_{i}}, m s g_{p} \in \tau_{x}\right)$ denotes the probability of sharing topic $\tau_{x}$ at time $t_{m}$ on the basis of $v_{i}$ 's past behaviours.

Thus, the attention degree of influence message $m s g_{p}$ in $v_{i}$ 's repository at time $t_{m}$ is formulated in Equation 9.

$$
P\left(m s g_{p} \mid R_{v_{i}}^{t_{m}}\right)=\frac{\sum_{r_{n} \in R_{v_{i}}^{t_{m}} \wedge r_{n} . m s g=m s g_{p}} \varphi\left(v_{r_{n}}, r_{n} . m s g, t_{r_{n}}\right)}{\sum_{r_{n} \in R_{v_{i}}^{t_{m}}} \varphi\left(v_{r_{n}}, r_{n} . m s g, t_{r_{n}}\right)},
$$

where $t_{r_{n}}=t_{m}-t_{n}, t_{n}$ denotes the time when the message $r_{n}$ arrives the repository.

According to $v_{i}$ 's historical records, the probability of sharing topic $\tau_{x}, m s g_{p} \in \tau_{x}$ at time $t_{m}$ can be derived from the weighted average of topic $\tau_{x}$ 's timeliness difference. Specifically, if $m s g_{p}$ has been posted when its timeliness degree $m s g_{p} . \varphi$ is low, this implies that the user is very interested in the topic of $m s g_{p}$ (i.e., $m s g_{p} . \tau$ ), and the message timeliness degree will not significantly impact the chances of posting such messages. Hence, $\left.P\left(\tau_{x} \mid H_{v_{i}}, m s g_{p} \in \tau_{x}\right)\right)$ is represented in Equation 10.

$$
\left.P\left(\tau_{x} \mid H_{v_{i}}, m s g_{p} \in \tau_{x}\right)\right)=\frac{\sum_{m s g_{p} \in \tau_{x}}\left(1-m s g_{p} \cdot \varphi\right)}{\sum_{m s g_{q} \in H_{v_{i}}}\left(1-m s g_{q} \cdot \varphi\right)}
$$

\subsection{Diffusion Process under the ATID}

Benefited from ABM, individual's features, behaviours and the local environment can be considered in the ATID. As the ATID is a decentralized influence propagation model, the diffusion algorithm under the ATID corresponds to an agent's response when accessing its repository. The diffusion process in the ATID is described in Algorithm 1.

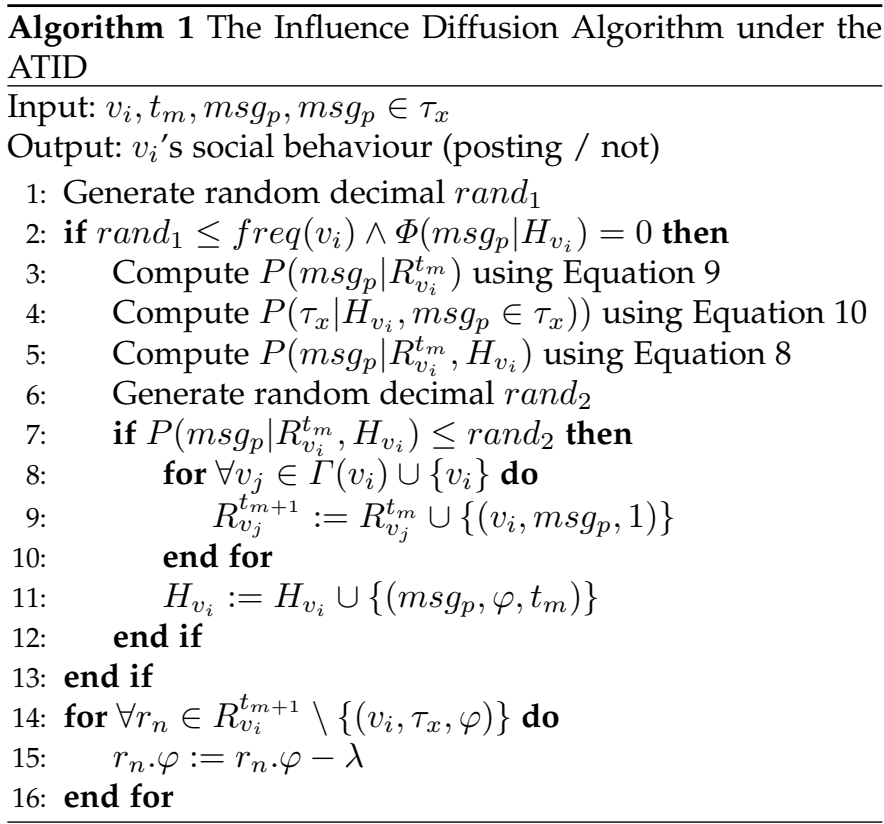

In Algorithm 1, the inputs incorporate user agent $v_{i}$, time $t_{m}$, the influence message $m s g_{p}$ and $m s g_{p}$ 's corresponding topic $\tau_{x}$; while the output is $v_{i}$ 's social behaviour, i.e., post $m s g_{p}$ at time $t_{m}$ or not. Line 2 checks the precondition of 
sharing $m s g_{p}$, where $\Phi\left(m s g_{p} \mid H_{v_{i}}\right)$ is an indicator function, which returns 0 if $m s g_{p}$ is not posted by $v_{i}$ before, and 1 otherwise. Lines 3-5 aim to compute the probability of posting $m s g_{p}$ by $v_{i}$ at $t_{m}$. Lines 8-11 update the repositories of agents in $v_{i}$ 's ego-network, as well as its own historical records. Lines 14-16 demonstrate that the message timeliness attenuation occurs in $v_{i}$ 's repository.

\subsection{The Timeliness Increase Heuristic (TIH) Algorithm}

There are some classic seed selection algorithms, such as degree-based, greedy, random and Degree Discount Heuristic (DDH) selections [4], [42]. These algorithms are developed based on either the node features or influence diffusion models. More specifically, degree-based approach identifies the influencers by considering the node degree. Greedy algorithm attempts to reach the maximum influence marginal gain in each selection, but it is not scalable. DDH extends the rank-based algorithm that once a node is selected, the degree of corresponding neighbours is deducted by one. Random selection does not follow any heuristics, which selects seeds randomly.

The rationale of developing $\mathrm{TIH}$ algorithm is clarified as follows. Since influence maintenance is newly proposed, no existing algorithms are exclusively designed for this problem. We attempt to leverage the classic approaches tailored from the influence maximization problem, and set further improvement of the performance as one of the future works. Based on the brief introduction of several stateof-the-art seeding algorithms in the traditional influence maximization problem, greedy selection is one of the fundamental algorithms, coming with a $(1-1 / e)$ approximation guarantee. This results from properties of monotonicity and sub-modularity that the spread function exhibits under some diffusion models [4]. Meanwhile, DDH is a simple and popular algorithm, which is developed based on the fact that many of the most central nodes may be clustered; thus, it is not necessary to target all of them [42].

Inspired by the key features of greedy algorithm and the intuitiveness of $\mathrm{DDH}$, we utilize the similar concepts to maintain an influence. Namely, the influence fading-out zone should be first targeted to achieve influence maintenance, and each selection is conducted based on the assumption that previous seed is selected. Therefore, TIH algorithm is presented in Algorithm 2. For selecting each seed, the TIH tends to search for the user $v^{*}$, who can bring the maximum message timeliness gain, which is calculated in Equations 11 and 12 .

$$
\begin{gathered}
v_{t_{m}}^{*}=\underset{v_{i}}{\operatorname{argmax}} \sum_{v_{j} \in\left\{v_{i}\right\} \cup \Gamma\left(v_{i}\right)} g\left(v_{j}, m s g_{p}, t_{m}\right) \\
g\left(v_{j}, m s g_{p}, t_{m}\right)=1-\varphi\left(v_{j}, m s g_{p}, t_{m}\right)
\end{gathered}
$$

In Equations 11 and 12, $g\left(v_{j}, m s g_{p}, t_{m}\right)$ denotes $v_{j}$ 's message timeliness gain if $v_{i}$ is selected as a seed. The selection of the next seed is based on the assumption that if previously identified seeds are selected. Thus, the TIH selection is described in Algorithm 2.

The inputs include the social network $G$, the number of seeds to be selected $k_{m}$, the time step $t_{m}$ and influence message $m s g_{p}$; the output is the selected seed set at $t_{m}$. Lines

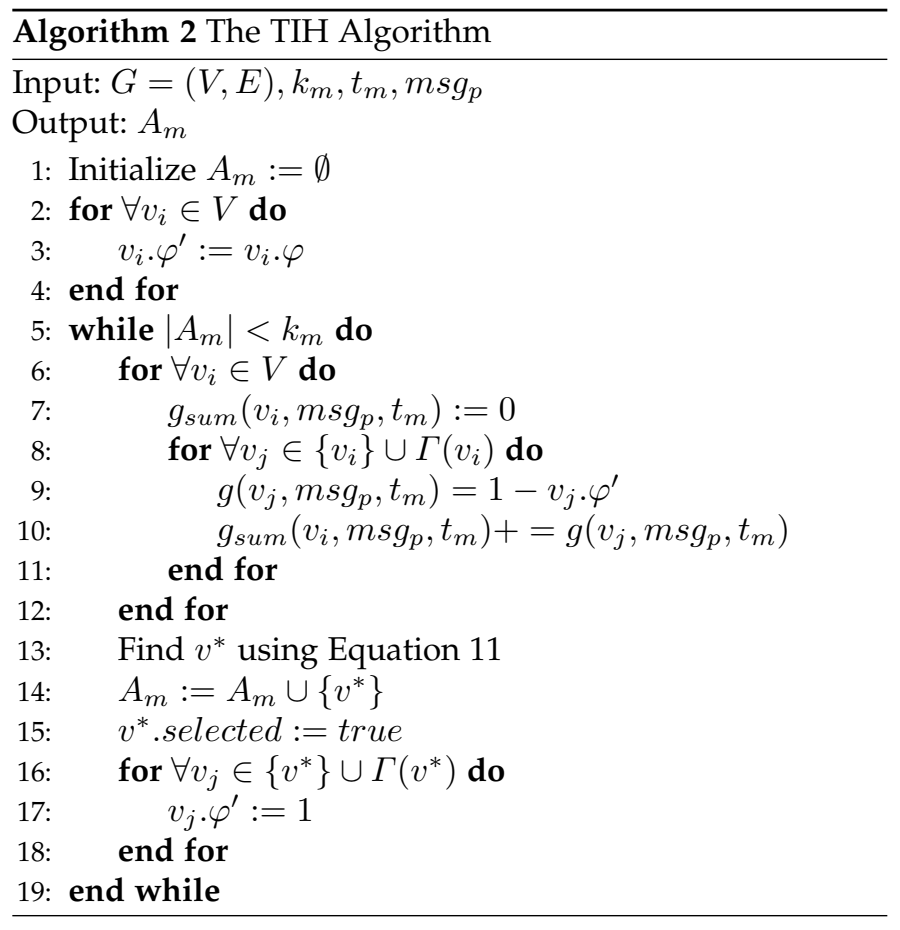

2-4 replicate all the user agents' current timeliness degree of $m s g_{p}$ to a temporary variable. Lines 6-11 calculate the global timeliness gain for all the users in $G$, in other words, this evaluates the influence impact of each individual. Lines 1213 aim to find the most 'beneficial' user. Lines 15-16 update the temporary timeliness variables of all the users in $v^{* \prime} \mathrm{s}$ ego network with the assumption that if $v^{*}$ is activated and selected as a seed. The worst-case time complexity of the TIH algorithm is determined by Lines $5-8$. As $k_{m}$ is a constant, the complexity is $O\left(n^{2}\right)$.

It can be seen that the seed set selected by TIH algorithm is the local optimal solution, following the heuristic that the largest timeliness fading-out zone should be firstly targeted. Moreover, the TIH demonstrates its advantages in maintaining the influence of a hypothesis message. 


$$
\begin{aligned}
\Delta \Omega & =\sum_{v_{j} \in\left\{v_{i}\right\} \cup \Gamma\left(v_{i}\right)} \sum_{t=t_{m}}^{t_{m+n}} \varphi^{\prime}\left(v_{j}, m s g_{p}, t\right)-\varphi\left(v_{j}, m s g_{p}, t\right) \\
& =\sum_{v_{j} \in\left\{v_{i}\right\} \cup \Gamma\left(v_{i}\right)}\left(\sum_{t=t_{m}}^{t_{m+n}} \varphi^{\prime}\left(v_{j}, m s g_{p}, t\right)-\sum_{t=t_{m}}^{t_{m+n}} \varphi\left(v_{j}, m s g_{p}, t\right)\right) \\
& =\sum_{v_{j} \in\left\{v_{i}\right\} \cup \Gamma\left(v_{i}\right)}\left(\sum_{i=0}^{n} e^{-i \cdot r}-\sum_{i=0}^{n} e^{-\left(m_{j}+i\right) \cdot r}\right) \\
& =\sum_{v_{j} \in\left\{v_{i}\right\} \cup \Gamma\left(v_{i}\right)}\left(\frac{1-e^{-(n+1) \cdot r}}{1-e^{-r}}-\frac{1-e^{-(n+1) \cdot r}}{1-e^{-r}} \cdot e^{-m_{j} \cdot r}\right) \\
& =\sum_{v_{j} \in\left\{v_{i}\right\} \cup \Gamma\left(v_{i}\right)} \frac{1-e^{-(n+1) \cdot r}}{1-e^{-r}} \cdot\left(1-e^{-m_{j} \cdot r}\right) \\
& =\frac{1-e^{-(n+1) \cdot r}}{1-e^{-r}} \sum_{v_{j} \in\left\{v_{i}\right\} \cup \Gamma\left(v_{i}\right)}\left(1-e^{-m_{j} \cdot r}\right) \\
& =\frac{1-e^{-(n+1) \cdot r}}{1-e^{-r}} \sum_{v_{j} \in\left\{v_{i}\right\} \cup \Gamma\left(v_{i}\right)}\left(1-\varphi\left(v_{j}, m s g_{p}, t_{m}\right)\right) \\
& =\frac{1-e^{-(n+1) \cdot r}}{1-e^{-r}} \sum_{v_{j} \in\left\{v_{i}\right\} \cup \Gamma\left(v_{i}\right)} g\left(v_{j}, m s g_{p}, t_{m}\right)
\end{aligned}
$$

Theorem 1. TIH is a kind of greedy algorithm.

Proof. Given current time step $t_{m}$, and $\varphi\left(v_{j}, m s g_{p}, t_{m}\right)=$ $e^{-m_{j} \cdot r}$, where $m_{j}$ denotes the time difference between when $m s g_{p}$ arrives and $t_{m}$. If node $v_{i}$ has been selected as a seed, the corresponding timeliness degree of node set $\left\{v_{j} \mid v_{j} \in\left\{v_{i}\right\} \cup \Gamma\left(v_{i}\right)\right\}$ is supposed to be reset back to 1 , i.e., $\varphi^{\prime}\left(v_{j}, m s g_{p}, t_{m}\right)=1$. Therefore, the incremental timeliness contribution of activating $v_{j}$, i.e., $\Delta \Omega$ can be derived using Equation 13.

In Equation 13, $n \in \mathbb{N}$, representing the difference between the total time steps and the current time step, and $e^{-m_{j} \cdot r}$ denotes the timeliness degree of a particular message in $v_{j}$ 's repository at $t_{m}$ according to Equation 1. It is obvious that $\frac{1-e^{-(n+1) \cdot r}}{1-e^{-r}}$ is a coefficient, $\sum_{v_{j} \in\left\{v_{i}\right\} \cup \Gamma\left(v_{i}\right)} g\left(v_{j}, m s g_{p}, t_{m}\right)$ exactly corresponds to the objective function of TIH algorithm in Equation 11. Therefore, $\mathrm{TIH}$ is a kind of greedy algorithm.

Lemma 2. Let $S$ be the seed set selected by TIH and $S^{*}$ be the seed set that maximizes $\Omega_{m s g_{p}}$. $\Omega_{m s g_{p}}(S)$ be the GCTD of msg with seed set $S$. Then $\Omega_{m s g_{p}}(S) \geq(1-1 / e) \cdot \Omega_{m s g_{p}}\left(S^{*}\right)$. In other words, the theoretical guarantee for $\mathrm{TIH}$ in the influence maintenance problem is $1-1 / e$.

Proof. Let $A$ be the initial seed set and $X=\left\langle v_{1}, v_{2}, \ldots, v_{h}\right\rangle$ be one of the paths activated by $A . f(A)$ represents the GCTD of $m s g_{p}$ caused by $A$. $f_{X}(A)$ denotes the GCTD accumulated by path $X$. Similar to the calculations in Equation 13, we have:

$$
f_{X}(A)=\sum_{j=1}^{h} \sum_{t=t_{m+j}}^{t_{m+n}} g\left(v_{j}, m s g_{p}, t\right)
$$

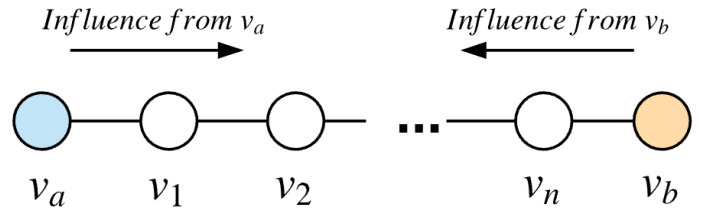

Fig. 2. One of the Overlapped Influence Diffusion Paths

, where $g\left(v_{j}, m s g_{p}, t_{m}\right)$ is defined in Equation 12, and $0 \leq$ $g\left(v_{j}, m s g_{p}, t\right) \leq 1$. It is easy to proof that $f_{X}(A)$ is submodular. Hence:

$$
f(A)=\sum_{\text {outcomes } x} \operatorname{Prob}|X| \cdot f_{X}(A)
$$

, which is also sub-modular since the non-negative linear combination of sub-modular functions is sub-modular. As we clarified in Theorem 1 that TIH is a kind of greedy algorithm. According to Theorem 2.4 in [4], we have $f(A) \geq$ $\left(1-\frac{1}{e}\right) f\left(A^{*}\right)$, where $A^{*}$ denotes the set that maximizes $f($. over all $k$-element sets.

Let $f(S)=\Omega_{m s g_{p}}(S)$, the lemma is proofed.

\subsection{Influence Maintenance Analysis}

We analyse the influence maintenance by considering the timeliness gain contributed by two seeds $v_{a}$ and $v_{b}$ under both scenarios, i.e., one-shot selection and multiple-time selection, where the time discrepancy of selecting both users is denoted by using $m_{0}$. In the former, no time discrepancy is presented, i.e., $m_{0}=0$, while in the latter, $m_{0} \neq 0$.

Suppose that enough time is given for the influence decay, i.e., $n \rightarrow \infty$, if any node is activated, the theoretical timeliness gain would be $1 /\left(1-e^{-r}\right)$ according to Equation 13. If all the influence-diffusion paths of active users fail to overlap with each other, the global timeliness gain of oneshot selection would be the same as that of the multiple-time selection. Whereas, in reality, this rarely happens. Therefore, we consider the situation when the influence-propagation paths cover same partial nodes with each other.

Suppose the influences disseminated from $v_{a}$ and $v_{b}$ can reach each other. In other words, path $\overrightarrow{v_{a, b}}=<$ $v_{a}, v_{1}, v_{2}, \ldots, v_{n}, v_{b}>$ exists in the network, which is illustrated in Figure 2. Moreover, for simplification purpose, we assume that the influence propagation probability remains the same.

For any node $v_{x}$ in path $\overrightarrow{v_{a, b}}$, the corresponding $\Delta \Omega$ is explicitly determined by the time discrepancy of the influences $\Delta T$ from two sources, $v_{a}$ and $v_{b}$, where $\Delta T$ is associated with the number of nodes in between, i.e., $n$ and the time difference in activating $v_{a}$ and $v_{b}$, i.e., $m_{0}$. Hence, values of influence maintenance related parameters are described in Table 2.

In Table 2, $T_{v_{a}}$ is an n-tuple, having a finite ordered list of $n$ elements, where each element denotes the time step when the influence initiated from $v_{a}$ arrives at the corresponding node, and the sequence implies the influence-diffusion path. Meanwhile, $\Delta T=\left(T_{v_{b}}-T_{v_{a}}\right)$, where each element indicates 
TABLE 2

Influence Maintenance Parameters

\begin{tabular}{lllll}
\hline & $v_{1}$ & $v_{2}$ & $\ldots$ & $v_{n}$ \\
\hline$T_{v_{a}}$ & $d_{0}$ & $d_{0}+1$ & $\ldots$ & $d_{0}+n-1$ \\
$T_{v_{b}}$ & $m_{0}+d_{0}+n-1$ & $m_{0}+d_{0}+n-2$ & $\ldots$ & $m_{0}+d_{0}$ \\
$\Delta T$ & $\left|m_{0}+n-1\right|$ & $\left|m_{0}+n-3\right|$ & $\ldots$ & $\left|m_{0}+n-(2 n-1)\right|$ \\
$\Delta \Omega$ & $\left|m_{0}+n-1\right|-1$ & $\left|m_{0}+n-3\right|-1$ & & $\left|m_{0}+n-(2 n-1)\right|-1$ \\
$\sum_{i=0} e^{-i r}$ & $\sum_{i=0}^{-i r} e^{-i r}$ & $\ldots$ & $\sum_{i=0} e^{-i r}$ \\
\hline
\end{tabular}

the absolute value of the difference between the elements in $T_{v_{b}}$ and $T_{v_{a}}$ at the same position.

If $\Delta T$ is odd, $\Delta T$ starts from $m_{0}+n-1$, decreasing by 2 further down the influence-diffusion path, and begins to increase by 2 for each hop when the value reaches 1 . Similarly, if $\Delta T$ is even, $\Delta T$ drops by 2 and then is added by 2 after reaching 0 . For example, given $n=6$, we can obtain the data in Table 3.

TABLE 3

Example: value variation of $\Delta T(\mathrm{n}=6)$

\begin{tabular}{ll}
\hline$m_{0}$ (even) & $\Delta T$ (odd) \\
\hline 0 & $(5,3,1,1,3,5)$ \\
2 & $(7,5,3,1,1,3)$ \\
4 & $(9,7,5,3,1,1)$ \\
6 & $(11,9,7,5,3,1)$ \\
\hline
\end{tabular}

\begin{tabular}{ll}
\hline$m_{0}$ (odd) & $\Delta T$ (even) \\
\hline 1 & $(6,4,2,0,2,4)$ \\
3 & $(8,6,4,2,0,2)$ \\
5 & $(10,8,6,4,2,0)$ \\
7 & $(12,10,8,6,4,2)$ \\
\hline
\end{tabular}

Apparently, in both scenarios where $\Delta T$ is even or odd, merely one different element can be seen when $m_{0}$ increases by 2 .

Lemma 3. $\forall k \in \mathbb{N}, \Delta \Omega\left(m_{0}=k+2\right)>\Delta \Omega\left(m_{0}=k\right)$.

Proof.

$$
\begin{aligned}
\Delta \Omega^{\prime} & =\Delta \Omega\left(m_{0}=k+2\right)-\Delta \Omega\left(m_{0}=k\right) \\
& =\sum_{i=0}^{|k+2+n-1|-1} e^{-i r}-\sum_{i=0}^{|k-n+1|-1} e^{-i r} \\
& =\sum_{i=|k-n+1|}^{|k+n+1|-1} e^{-i r}>0,\{n, k\} \in \mathbb{N}, n \geq 1
\end{aligned}
$$

According to Lemma $3, \forall k \in \mathbb{N}$, we have:

$$
\begin{aligned}
& \Delta \Omega\left(m_{0}=0\right)<\Delta \Omega\left(m_{0}=2\right)<\ldots<\Delta \Omega\left(m_{0}=2 k\right) \\
& \Delta \Omega\left(m_{0}=1\right)<\Delta \Omega\left(m_{0}=3\right)<\ldots<\Delta \Omega\left(m_{0}=2 k+1\right)
\end{aligned}
$$

Theorem 4. Multiple-time selection maintains a particular influence more effectively than that of one-shot selection.

Proof. Based on Equation 14, we only need to proof $\Delta \Omega\left(m_{0}=1\right)>\Delta \Omega\left(m_{0}=0\right)$. Assume that the path length between two active nodes has an equal chance to be even or odd. In other words, $P(n=2 h)=P(n=2 k+1)$, where $k, h \in \mathbb{N}$. The values of $\Delta T$ and $\Delta \Omega$ under different parameters are listed and compared in Tables 4 and 5 . Then we can obtain Equations 15 and 16.

$$
\begin{aligned}
& \Delta \Omega\left(n=2 k+1, m_{0}=1\right)-\Delta \Omega\left(n=2 h, m_{0}=0\right) \\
& =2 \sum_{i=0}^{2 h} e^{-i r}+\ldots+2 \sum_{i=0}^{2 k-2} e^{-i r}+\sum_{i=0}^{2 k} e^{-i r} \\
& \Delta \Omega\left(n=2 h, m_{0}=1\right)-\Delta \Omega\left(n=2 k+1, m_{0}=0\right) \\
& =-\left(\sum_{i=0}^{2 h-1} e^{-i r}+2 \sum_{i=0}^{2 h+1} e^{-i r}+\ldots+2 \sum_{i=0}^{2 k-1} e^{-i r}\right)
\end{aligned}
$$

Suppose $h>k$, then by adding Equation 15 to Equation 16, we can obtain:

$$
\begin{aligned}
& \left(\Delta \Omega\left(n=2 h, m_{0}=1\right)+\Delta \Omega\left(n=2 k+1, m_{0}=1\right)\right) \\
& -\left(\Delta \Omega\left(n=2 k+1, m_{0}=0\right)+\Delta \Omega\left(n=2 h, m_{0}=0\right)\right) \\
= & e^{-2 h r}+\ldots+e^{-2(2 k-2) r}+e^{-2 k r} \\
& -\left(e^{-(2 h+1) r}+\ldots+e^{-(2 k-1) r}\right) \\
= & \left(e^{-2 h r}-e^{-(2 h+1) r}\right)+\ldots \\
& +\left(e^{-(2 k-2) r}-e^{-(2 k-1) r}\right)+e^{-2 k r} \\
& >e^{-2 k r}>0
\end{aligned}
$$

The same proof can be applied when $h \leq k$. Therefore, $\Delta \Omega\left(m_{0}=1\right)>\Delta \Omega\left(m_{0}=0\right)$.

\section{EXPERIMENTS AND ANALYSIS}

We conducted three major experiments for this research work. The first one aims to compare the difference in influence impact between one-shot and multiple-time investment. The second experiment evaluates the performance of the TIH algorithm. In the third experiment, we further compare one-shot selection against multiple-time selection by exploring the variations of selected seeds based on the ATID model.

\subsection{Experiment Setup}

Datasets. In the experiments, the following three datasets are used.

- Ego-Facebook ${ }^{5}$ dataset, collected by McAuley et al. using a Facebook application, which is archived in Stanford Large Network Dataset Collection [43]. It contains profile and network data from 10 egonetworks, consisting of 193 circles, 4,039 users and 88,234 edges.

5. http://snap.stanford.edu/data/egonets-Facebook.html 
TABLE 4

Value Comparison for $\Delta T$ and $\Delta \Omega(\mathrm{n}=2 \mathrm{~h})$

\begin{tabular}{|c|c|c|c|c|c|c|}
\hline & $v_{1}$ & $\ldots$ & $v_{h}$ & $v_{h+1}$ & $v_{h+2}$ & $v_{2 h}$ \\
\hline$\Delta T\left(n=2 h, m_{0}=0\right)$ & $2 h-1$ & & 1 & 1 & 3 & $2 h-1$ \\
\hline$\Delta \Omega\left(n=2 h, m_{0}=0\right)$ & $\sum_{i=0}^{2 h-2} e^{-i r}$ & & $\sum_{i=0}^{0} e^{-i r}$ & $\sum_{i=0}^{0} e^{-i r}$ & $\sum_{i=0}^{2} e^{-i r}$ & $\sum_{i=0}^{2 h-2} e^{-i r}$ \\
\hline$\Delta T\left(n=2 h, m_{0}=1\right)$ & $2 h$ & & 2 & 0 & 2 & $2 h-2$ \\
\hline$\Delta \Omega\left(n=2 h, m_{0}=1\right)$ & $\sum_{i=0}^{2 h-1} e^{-i r}$ & & $\sum_{i=0}^{1} e^{-i r}$ & 0 & $\sum_{i=0}^{1} e^{-i r}$ & $\sum_{i=0}^{2 h-3} e^{-i r}$ \\
\hline
\end{tabular}

TABLE 5

Value Comparison for $\Delta T$ and $\Delta \Omega(\mathrm{n}=2 \mathrm{k}+1)$

\begin{tabular}{|c|c|c|c|c|c|c|c|}
\hline & $v_{1}$ & $\ldots$ & $v_{k}$ & $v_{k+1}$ & $v_{k+2}$ & $\ldots$ & $v_{2 k+1}$ \\
\hline$\Delta T\left(n=2 k+1, m_{0}=0\right)$ & $2 k$ & & 2 & 0 & 2 & & $2 k$ \\
\hline$\Delta \Omega\left(n=2 k+1, m_{0}=0\right)$ & $\sum_{i=0}^{2 k-1} e^{-i r}$ & & $\sum_{i=0}^{1} e^{-i r}$ & 0 & $\sum_{i=0}^{1} e^{-i r}$ & & $\sum_{i=0}^{2 k-1} e^{-i r}$ \\
\hline$\Delta T\left(n=2 k+1, m_{0}=1\right)$ & $2 k+1$ & & 3 & 1 & 1 & & $2 k-1$ \\
\hline$\Delta \Omega\left(n=2 k+1, m_{0}=1\right)$ & $\sum_{i=0}^{2 k} e^{-i r}$ & & $\sum_{i=0}^{2} e^{-i r}$ & $\sum_{i=0}^{0} e^{-i r}$ & $\sum_{i=0}^{0} e^{-i r}$ & & $\sum_{i=0}^{2 k-2} e^{-i r}$ \\
\hline
\end{tabular}

- Email-Enron ${ }^{6}$ dataset, which covers all the email communication. It has been posted to the web by the Federal Energy Regulatory Commission [44]. The Enron email network has 36,692 nodes and 367,662 Edges. To diminish the computing time, we capture a sub-graph with 10k nodes for the experiment.

- Wiki-Vote $\mathbf{y}^{7}$ dataset, which incorporates administrator elections and votes history data from 3 January 2008. There are 2,794 elections with 103,663 total votes and 7,066 users participating in the elections. Nodes refer to Wikipedia users and edges represent votes from one user to another [45].

System Setup. We simulate the social context by creating a number of user agents based on the public datasets. Each user agent manages its local information, including a friendship list, a repository and historical records. We assume a hypothesis influence message is supposed to be maintained and each agent has a different tendency of posting this message. In the meanwhile, the reporting agent is responsible for monitoring the entire multi-agent system and collecting global information. The system has three types of states as follows:

- Evolve: user agents perform actions, incorporating accessing the repository, reading the message and making decisions (share the post or not) based on both past experiences and timeliness degrees.

- Pause: the entire system pauses, and stops functioning temporarily. This state allows seed selection algorithms to identify influential users and select seeds based on the current network status. In other words, further investment happens at this point. The system evolution resumes as soon as the seed selection is completed.

- Stop: All the user agents decompose, and the system terminates.

6. https://snap.stanford.edu/data/email-Enron.html

7. https://snap.stanford.edu/data/wiki-Vote.html
TABLE 6

Experiment Parameters

\begin{tabular}{ll}
\hline Parameter & Value(s) \\
\hline Fixed time steps for seed selections & 100 \\
Fixed time steps in total & 150 \\
$\begin{array}{l}\text { Number of seeds to be selected for each } \\
\text { selection points }\end{array}$ & $25,5,1$ \\
$\begin{array}{l}\text { The interval (time steps) of seed } \\
\text { selection }\end{array}$ & $100,20,4$ \\
$\begin{array}{l}\text { Seed set size } \\
\text { attenuation constant } r\end{array}$ & 25 \\
$\begin{array}{l}\text { General action frequency of user agents } \\
\text { (times per second) }\end{array}$ & 0.1 \\
\hline
\end{tabular}

By setting up the system, the parameters for the experiments are given in Table 6. We assume that the observations of network evolution are within a fixed interval, and the same amount of seeds are supposed to be selected at each seed selection point. To reduce the bias of measuring the performance of different strategies, additional time steps, i.e., 50 time steps in our experiments, are given after the final seed selection for the influence dissemination and attenuation. Furthermore, the budget is limited, in other words, the seed set size is limited. The overall action frequency of user agents controls the speed of network evolution.

Evaluation Metrics. As introduced in Section 3.4, three major evaluation metrics are taken into consideration, i.e., GTD, GCTD and GAC, which have been explained and formulated in Equations 4, 5 and 6, respectively. GCTD and GAC were applied in both Experiment 1 and Experiment 2 for comparing the performance of different selection strategies. GTD has been mainly utilized in Experiment 1 for tracking the variation of timeliness degree of a particular influence message in different time steps. In Experiment 3 , some distance indices were facilitated to measure the variation of seed sets. 


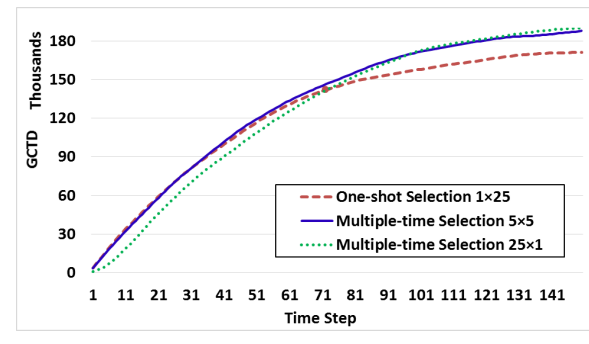

Fig. 3. Rank-based (GCTD)

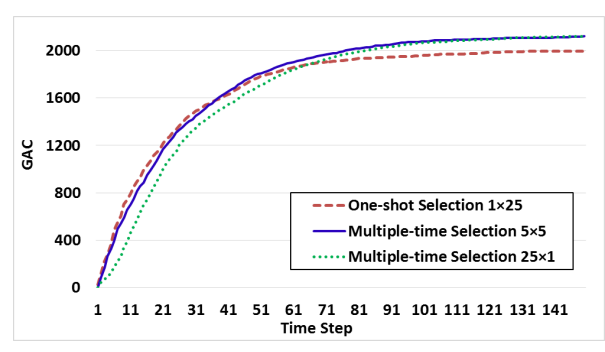

Fig. 6. Rank-based (GAC)

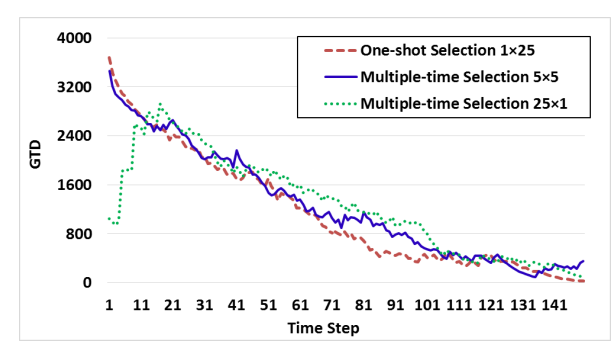

Fig. 9. Rank-based (GTD)

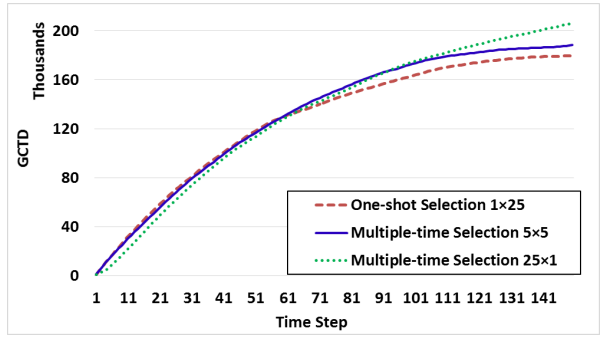

Fig. 4. DDH Selection (GCTD)

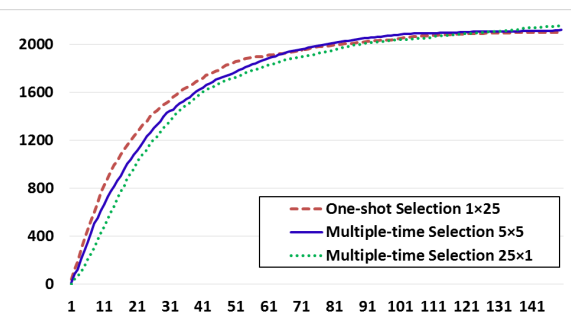

Fig. 7. DDH Selection (GAC)

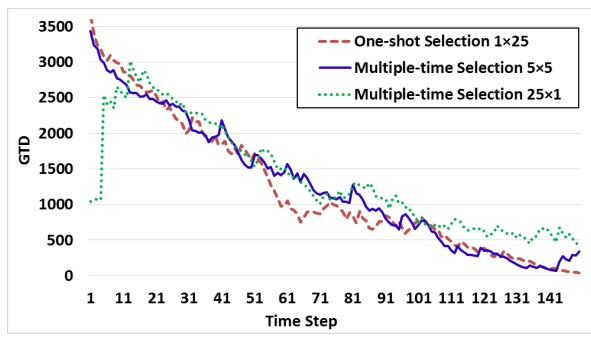

Fig. 10. DDH Selection (GTD)

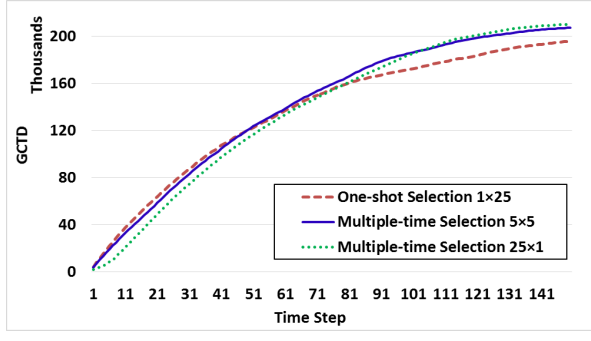

Fig. 5. TIH Selection (GCTD)

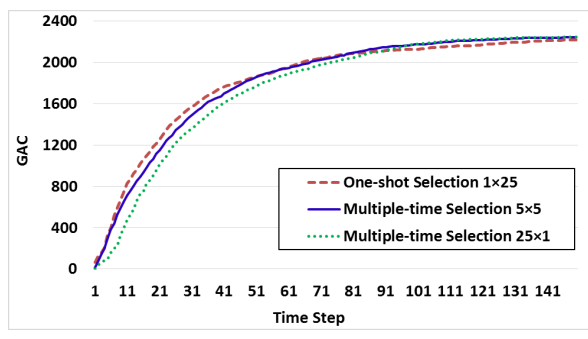

Fig. 8. TIH Selection (GAC)

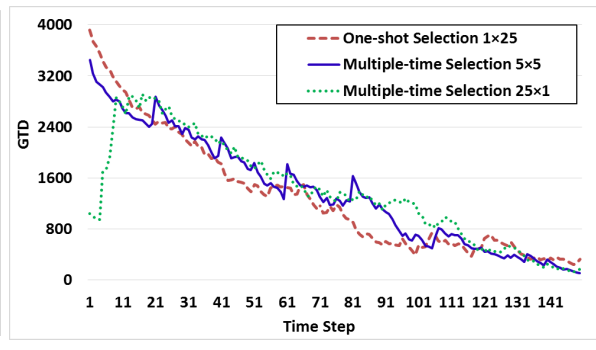

Fig. 11. TIH Selection (GTD)

\subsection{Experiment 1: One-shot vs multiple-time selection}

Experiment 1 compares one-shot investment against the multiple-time by facilitating different seed selection algorithms, i.e., rank-based, DDH and the TIH selection. In this experiment, the Ego-Facebook dataset is applied for the explorations. The notations of selection approaches are listed in Table 7.

TABLE 7

Notations of Selections

\begin{tabular}{ll}
\hline Notation & Meaning \\
\hline $1 \times 25$ & One-shot selection, 25 seeds \\
$5 \times 5$ & 5-time selection, select 5 each time \\
$25 \times 1$ & 25-time selection, select 1 each time \\
\hline
\end{tabular}

As we can observe from Figures 3, 4 and 5 that multipletime selections can produce higher GCTD. The gap between one-shot selection and multiple-time selection turns out to be evident over time. $5 \times 5$ and $25 \times 1$ give pretty close performance, but $25 \times 1$ shows slightly better, especially after 100 time steps when the selections are completed. By comparing the GAC in Figures 6, 7 and 8, the multiple-time selection also outperforms the one-shot selection. One-shot selection demonstrates a rapid influence activation coverage, but unfortunately it loses the leading position halfway.

Based on the results in Figures 3 - 8, we can observe that with the same budget, increasing the frequency of investments generally carries out higher GCTD and GAC, subject to additional time for influence diffusion and attenuation provided. This is due to the reason that the last-round investment in multiple-time selections is not supposed to give much credit without additional time for influence spread and decay. The results also explicitly reveal that multipletime selections target the reward in the long-run, but may yield short-term performance. If an organisation intends to maintain an influence by considering both effectiveness and time required for GCTD to reach a certain level, selection strategies with extremely high frequencies $(25 \times 1$ in our experiment) may not be advocated, since it takes longer time to reach the maximum GCTD. Whereas, $5 \times 5$ balances the trade-off between time and GCTD, which is a better option under such a scenario. The same rule also applies to GAC.

To drill down into the details, we explore timeliness variations of the influence message after adopting different selection strategies in Figures 9, 10 and 11. One-shot selection has the highest starting point, but it declines faster than that of $5 \times 5$ and generally falls behind the others after around 20 time steps. Obvious spikes can be observed in multiple-time selections and appear to be more prominent 
in the TIH $5 \times 5$. Whereas, $25 \times 1$ demonstrates a different pattern. It climbs to the peak point, which is higher than that of the other two selection approaches, then falls gradually. The organisation expects a sharp upward trend after each investment. However, this is not guaranteed based on the results. For example, no obvious increase can be observed at time steps 60 and 20 of rank-based $5 \times 5$ and DDH $5 \times 5$, respectively. In contrast, the TIH $5 \times 5$ sees an evident spike after each investment.

\subsection{Experiment 2: The TIH Seed Selection Evaluation}

Experiment 2 aims to evaluate the performance of the $\mathrm{TIH}$ algorithm. We compare the proposed TIH algorithm against state-of-the-art algorithms. Since the diffusion model is probabilistic based, the results are obtained by averaging multiple trials. To reduce the bias, we evaluate the $\mathrm{TIH}$ algorithm by using the three datasets mentioned previously, i.e., Ego-Facebook, Email-Enron and Wiki-Vote.

The experimental results are demonstrated in Table 8. It can be seen that the TIH outperforms the others in all the three datasets. By using any selection strategy, the TIH performs the best in terms of GCTD and GAC.

Another intriguing finding from the experimental results is concerning the relationship between GCTD and GAC. More specifically, given the same budget, GCTD rises with the increment of selection trails. In general, GAC gains when GCTD increases. However, by adopting rank-based $25 \times 1$, the GAC yields that of the $5 \times 5$, though GCTD rises. This phenomenon implicitly shows that the outcome of influence maintenance is not always in accordance with that of the influence maximization. Whereas, the relationship between GCTD and GAC tends to be affected by the applied business strategies. In other words, the strategies created for long-term marketing can possibly suppress the short-term growth of the product adoptions.

\subsection{Experiment 3: Seed Set Variation Analysis}

With the same budget, different selection approaches inevitably produce different seed sets. To understand the outcome of various strategies, in this experiment, we further compare one-shot selection against multiple-time selection by exploring the variations of selected seeds based on the ATID model. The TIH algorithm has been applied for the seeding procedures in three social networks mentioned previously.

Three evaluation metrics are adopted for measuring the distance (referring to variation or dissimilarity) between any two seed sets, i.e., Jaccard distance $d_{j c d}\left(A_{1}, A_{2}\right)$, Dice dissimilarity $d_{d i c}\left(A_{1}, A_{2}\right)$ and sequential distance considering the index of the elements $d_{s q c}\left(A_{1}, A_{2}\right)$, which are formulated in Equations 18, 19 and 20, respectively. In these three equations, $A_{1}$ and $A_{2}$ denote two different seed sets, having the same cardinality, i.e., $A_{1} \neq A_{2},\left|A_{1}\right|=\left|A_{2}\right| . I\left(c \mid A_{1}\right)$ refers to the index of element $c$ in set $A_{1}$.

$$
\begin{gathered}
d_{j c d}\left(A_{1}, A_{2}\right)=1-\frac{\left|A_{1} \cap A_{2}\right|}{\left|A_{1} \cup A_{2}\right|} \\
d_{d i c}\left(A_{1}, A_{2}\right)=1-\frac{2\left|A_{1} \cap A_{2}\right|}{\left|A_{1}\right|+\left|A_{2}\right|}
\end{gathered}
$$

$$
d_{s q c}\left(A_{1}, A_{2}\right)=\frac{1}{\left|A_{1}\right|}\left(\sum_{c \in A_{1} \cap A_{2}} \frac{\left|I\left(c \mid A_{1}\right)-I\left(c \mid A_{2}\right)\right|}{\left|A_{1}\right|}+\left|A_{1} \backslash A_{2}\right|\right)
$$

As the influence diffusion appears to be probabilisticbased, different sets of the nodes could be selected by using the same algorithm. To reduce the bias, results are averaged over multiple trials. Figure 12 compares the variations of the seed sets produced by using different strategies. It explicitly shows that the seed-set variations between oneshot selection and multiple-time selection appear to be more prominent when having a higher frequency of selections. Two multiple-time selection approaches, i.e., $5 \times 5$ and $25 \times 1$, share a larger overlapping seeds than that of oneshot selection.

To investigate the correlations between network properties and seed-set variations, we list the detailed results in Table 9, where "Average Path Length" (APL) refers to the average number of steps along the shortest paths for all possible pairs of nodes. APL is one of the key metrics to measure the transitivity of the network [46]. A shorter APL generally indicates that less time is required for any influence travelling from one node to another.

It can be seen from Table 9 that a greater average path length corresponds to a higher seed-set variation. The reason behind is that in shorter APL networks, influences become relatively easier to reach any node, thus $e^{-r \cdot(m-b)}$ in Equation 1 appears to be lower as $b$ shrinks. Subsequently, timeliness gain turns out to be less prominent. Therefore, based on Equations 11 and 12, the TIH algorithm has a higher chance to carry out similar seed sets under such circumstances.

\subsection{Discussion}

We simulated a social environment and the process of influence maintenance in a social network. Through the experiments, we demonstrated the advantages of applying ATID to model the influence propagation process. Two critical factors required by the influence maintenance can be presented clearly in the ATID, i.e., the temporal feature of the social network and the status of a particular influence. Furthermore, the seed-set variations are compared after applying different selection approaches. We also evaluate the effectiveness of various seed-selection algorithms in maintaining an influence. The TIH algorithm surpasses some selected traditional selection algorithms by using three different datasets.

More importantly, three empirical laws can be drawn from the experimental results. (1) Given the same budget, the multiple-time investment is generally more beneficial for achieving the long-lasting influence of a particular product than that of the one-shot investment. (2) Influence maintenance is not always in accordance with that of the influence maximization. In other words, sustaining a longterm impact of a particular influence cannot ensure a large fraction of activation coverage; the long-term marketing strategies may hinder the profit spikes. (3) Seed-set variation is not only associated with the frequency of selections, but also affected by the network property. A greater average path length of social networks leads to a higher seed-set variations. 
TABLE 8

Seed Selection Performance Comparison

\begin{tabular}{cccccc}
\hline Social Network & Algorithm & Metrics & $\begin{array}{c}\text { One-shot } \\
\text { Selection } 1 \times 25\end{array}$ & $\begin{array}{c}\text { Multiple-time } \\
\text { Selection } 5 \times 5\end{array}$ & $\begin{array}{c}\text { Multiple-time } \\
\text { Selection } 25 \times 1\end{array}$ \\
\hline Ego-Facebook & TIH & GCTD & $\mathbf{1 9 5 , 9 5 1}$ & $\mathbf{2 0 7 , 1 1 5}$ & $\mathbf{2 0 9 , 6 7 5}$ \\
Ego-Facebook & TIH & GAC & $\mathbf{2 , 2 2 2}$ & $\mathbf{2 , 2 4 2}$ & $\mathbf{2 , 2 4 9}$ \\
Ego-Facebook & RANK & GCTD & 170,966 & 187,696 & 189,968 \\
Ego-Facebook & RANK & GAC & 1,996 & 2,124 & 2,118 \\
Ego-Facebook & DDH & GCTD & 181,994 & 190,653 & 205,774 \\
Ego-Facebook & DDH & GAC & 2,097 & 2,151 \\
Ego-Facebook & Random & GCTD & 168,733 & 175,899 & 188,300 \\
Ego-Facebook & Random & GAC & 1,889 & 1,988 & 2,003 \\
\hline Email Eron & TIH & GCTD & $\mathbf{3 4 1 , 4 1 8}$ & $\mathbf{3 5 8 , 7 2 2}$ & $\mathbf{3 8 4 , 8 6 1}$ \\
Email Eron & TIH & GAC & $\mathbf{4 , 3 0 7}$ & $\mathbf{4 , 4 4 5}$ & $\mathbf{4 , 3 3 1}$ \\
Email Eron & RANK & GCTD & 328,026 & 352,744 & 362,992 \\
Email Eron & RANK & GAC & 4,082 & 4,391 & 4,365 \\
Email Eron & DDH & GCTD & 338,803 & 355,452 & 373,218 \\
Email Eron & DDH & GAC & 4,227 & 4,255 & 4,492 \\
Email Eron & Random & GCTD & 324,994 & 337,380 & 338,269 \\
Email Eron & Random & GAC & 4,181 & 4,189 & 4,196 \\
\hline Wiki Vote & TIH & GCTD & $\mathbf{2 5 4 , 7 1 0}$ & $\mathbf{2 6 7 , 8 1 0}$ & $\mathbf{2 7 2 , 2 9 2}$ \\
Wiki Vote & TIH & GAC & $\mathbf{2 , 8 6 8}$ & $\mathbf{3 , 0 0 1}$ & $\mathbf{2 , 9 5 3}$ \\
Wiki Vote & RANK & GCTD & 247,659 & 264,417 & 267,213 \\
Wiki Vote & RANK & GAC & 2,826 & 2,944 & 2,878 \\
Wiki Vote & DDH & GCTD & 249,977 & 265,950 & 270,906 \\
Wiki Vote & DDH & GAC & 2,843 & 2,954 & 2,829 \\
Wiki Vote & Random & GCTD & 247,626 & 253,349 & $2,89,599$ \\
Wiki Vote & Random & GAC & $\mathbf{2 , 8 1 3}$ & 2,843 & 24 \\
\hline
\end{tabular}

TABLE 9

Network Properties and Seed Sets Variations

\begin{tabular}{|c|c|c|c|c|c|c|c|c|c|c|}
\hline \multirow[t]{2}{*}{ Dataset } & \multirow{2}{*}{$\begin{array}{c}\text { Average } \\
\text { Path Length }\end{array}$} & \multicolumn{3}{|c|}{$\begin{array}{l}\text { one-shot selection vs. } \\
\text { multiple-time selection } 5 \times 5\end{array}$} & \multicolumn{3}{|c|}{$\begin{array}{l}\text { one-shot selection vs. } \\
\text { multiple-time selection } 25 \times 1\end{array}$} & \multicolumn{3}{|c|}{$\begin{array}{r}\text { multiple-time selection } 5 \times 5 \\
\text { vs. multiple-time selection } 25 \times\end{array}$} \\
\hline & & Jaccard & Dice & Sequence & Jaccard & Dice & Sequence & Jaccard & Dice & Sequence \\
\hline Email Enron & & 0.442 & 0.284 & 0.365 & 0.498 & 0.332 & 0.424 & 0.094 & 0.051 & 0.113 \\
\hline Wiki Vote & 3.247 & 0.622 & 0.452 & 0.540 & 0.730 & 0.576 & 0.650 & 0.424 & 0.270 & 0.394 \\
\hline Ego-Facebook & 3.693 & 0.768 & 0.624 & 0.631 & 0.792 & 0.656 & 0.668 & 0.477 & 0.315 & 0.383 \\
\hline
\end{tabular}

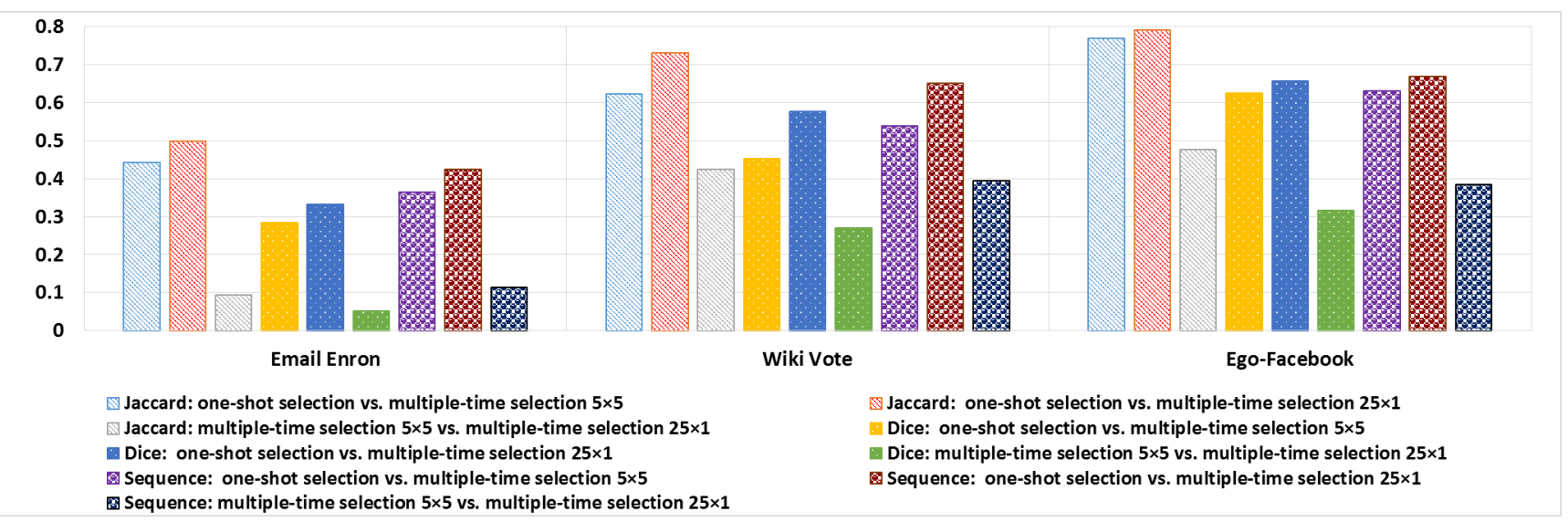

Fig. 12. Seed Set Variation Comparison under Different Strategies 


\section{Conclusions ANd Future Work}

In this paper, we systematically studied the influence maintenance problem, which targets the long-term and sustainable business goals. To the best of our knowledge, this paper is the first full research work that characterizes the influence maintenance in social networks. The distributed influence diffusion model, i.e., the ATID, presented in this article can also pave the way in exploring influence propagation social pheromone, since it concentrates on modelling the agent's personalized traits and behaviours, tracking the temporal feature of a social network, as well as the status of influence messages. Many features of both individuals and influences can be enabled in the ATID when analysing the social influence diffusion phenomenon. We have also proposed a novel seed selection algorithm, i.e., the TIH, which is capable of maintaining long-term influence effectively. Extensive experiments are conducted, and the empirical results show that the proposed model is capable of enhancing long-term influence. Given the same budget and limited time frame, multiple-time investment is superior to one-shot investment in terms of influence maintenance. Moreover, the experimental results also explicitly show that the TIH performs better than the other traditional selection algorithms by considering GCTD and GAC. We believe that our findings can shed light on the understanding of influence maintenance for long-term marketing.

In the future, we plan to free up the assumptions. Specifically, we will try to explore the solutions for the situations, where (1) the time step is not fixed; (2) for each investment, the seed set size is not fixed; and (3) the seed selection point can be a variant.

\section{REFERENCES}

[1] John C Turner. Social influence. Thomson Brooks/Cole Publishing Co, 1991.

[2] Bertram H Raven. Social influence and power. Technical report, CALIFORNIA UNIV LOS ANGELES, 1964.

[3] Young Kim and Jaideep Srivastava. Impact of social influence in ecommerce decision making. In Proceedings of the ninth international conference on Electronic commerce, pages 293-302. ACM, 2007.

[4] David Kempe, Jon Kleinberg, and Éva Tardos. Maximizing the spread of influence through a social network. In Proceedings of the ninth ACM SIGKDD international conference on Knowledge discovery and data mining, pages 137-146, Washington, DC, USA, 2003. ACM.

[5] Alex Valencia. Short-term vs. long-term online marketing, August 2013. [Online; posted 22-August-2013].

[6] Launch Marketing. What are your short-and long-term marketing strategies?, August 2015. [Online; posted 26-August-2015].

[7] Jennifer Bender et al. Best practices for investment risk management. Journal of Financial Transformation, 28:37-43, 2010.

[8] D Aaker, DA Aaker, and A Biel. Are brand equity investments really worthwhile. Brand equity and advertising: Advertising's role in building strong brands, pages 333-341, 1993.

[9] Weihua Li, Quan Bai, Tung Doan Nguyen, and Minjie Zhang. Agent-based influence maintenance in social networks. In Proceedings of the 16th Conference on Autonomous Agents and MultiAgent Systems (Extended Abstract), pages 1592-1594. International Foundation for Autonomous Agents and Multiagent Systems, 2017.

[10] Pedro Domingos and Matt Richardson. Mining the network value of customers. In Proceedings of the seventh ACM SIGKDD international conference on Knowledge discovery and data mining, pages 57-66. ACM, 2001.

[11] Daniel Golovin and Andreas Krause. Adaptive submodularity: Theory and applications in active learning and stochastic optimization. Journal of Artificial Intelligence Research, 42:427-486, 2011.
[12] Noga Alon, Iftah Gamzu, and Moshe Tennenholtz. Optimizing budget allocation among channels and influencers. In Proceedings of the 21st international conference on World Wide Web, pages 381-388. ACM, 2012.

[13] Tasuku Soma, Naonori Kakimura, Kazuhiro Inaba, and Ken-ichi Kawarabayashi. Optimal budget allocation: Theoretical guarantee and efficient algorithm. In Proceedings of The 31st International Conference on Machine Learning, pages 351-359, 2014.

[14] Daisuke Hatano, Takuro Fukunaga, and Ken-ichi Kawarabayashi. Adaptive budget allocation for maximizing influence of advertisements. In Proceedings of the 25th International Joint Conference on Artificial Intelligence IJCAI-16, pages 3600-3608, 2016.

[15] Yu Yang, Xiangbo Mao, Jian Pei, and Xiaofei He. Continuous influence maximization: What discounts should we offer to social network users? In Proceedings of the 2016 International Conference on Management of Data, pages 727-741. ACM, 2016.

[16] Manuel Gomez Rodriguez and Bernhard Schölkopf. Influence maximization in continuous time diffusion networks. arXiv preprint arXiv:1205.1682, 2012.

[17] Charles M Macal and Michael J North. Agent-based modeling and simulation. In Proceedings of the Winter Simulation Conference, pages 86-98. IEEE, 2009.

[18] Eric Bonabeau. Agent-based modeling: Methods and techniques for simulating human systems. Proceedings of the National Academy of Sciences, 99(suppl 3):7280-7287, 2002.

[19] Konstantin Kutzkov, Albert Bifet, Francesco Bonchi, and Aristides Gionis. Strip: stream learning of influence probabilities. In Proceedings of the 19th ACM SIGKDD international conference on Knowledge discovery and data mining, pages 275-283. ACM, 2013.

[20] Karthik Subbian, Charu C Aggarwal, and Jaideep Srivastava. Querying and tracking influencers in social streams. In Proceedings of the Ninth ACM International Conference on Web Search and Data Mining, pages 493-502. ACM, 2016.

[21] Yanhao Wang, Qi Fan, Yuchen Li, and Kian-Lee Tan. Real-time influence maximization on dynamic social streams. Proceedings of the VLDB Endowment, 10(7):805-816, 2017.

[22] Qiyao Wang, Yuehui Jin, Zhen Lin, Shiduan Cheng, and Tan Yang. Influence maximization in social networks under an independent cascade-based model. Physica A: Statistical Mechanics and its Applications, 444:20-34, 2016.

[23] Amit Goyal, Francesco Bonchi, and Laks V.S. Lakshmanan. Learning influence probabilities in social networks. In Proceedings of the Third ACM International Conference on Web Search and Data Mining, WSDM '10, pages 241-250, New York, NY, USA, 2010. ACM.

[24] Jie Tang, Jimeng Sun, Chi Wang, and Zi Yang. Social influence analysis in large-scale networks. In Proceedings of the 15th ACM SIGKDD international conference on Knowledge discovery and data mining, pages 807-816. ACM, 2009.

[25] Hao Ma, Haixuan Yang, Michael R Lyu, and Irwin King. Mining social networks using heat diffusion processes for marketing candidates selection. In Proceedings of the 17th ACM conference on Information and knowledge management, pages 233-242. ACM, 2008.

[26] Yi-Cheng Chen, Wen-Yuan Zhu, Wen-Chih Peng, Wang-Chien Lee, and Suh-Yin Lee. Cim: community-based influence maximization in social networks. ACM Transactions on Intelligent Systems and Technology (TIST), 5(2):25, 2014.

[27] Quan Bai, Minjie Zhang, and Haijun Zhang. A colored petri net based strategy for multi-agent scheduling. In Rational, Robust, and Secure Negotiation Mechanisms in Multi-Agent Systems, 2005, pages 3-10. IEEE, 2005.

[28] Dayong Ye, Minjie Zhang, and Athanasios V Vasilakos. A survey of self-organization mechanisms in multiagent systems. 2016.

[29] Yichuan Jiang and JC Jiang. Diffusion in social networks: A multiagent perspective. IEEE Transactions on Systems, Man, and Cybernetics: Systems, 45(2):198-213, 2015.

[30] Zhenpeng $\mathrm{Li}$ and Xijin Tang. From global polarization to local social mechanisms: A study based on abm and empirical data analysis. In Smart Modeling and Simulation for Complex Systems, pages 29-40. Springer, 2015.

[31] Peter-Paul van Maanen and Bob van der Vecht. An agent-based approach to modeling online social influence. In Proceedings of the 2013 IEEE/ACM International Conference on Advances in Social Networks Analysis and Mining, pages 600-607. ACM, 2013.

[32] Weihua Li, Quan Bai, and Minjie Zhang. Agent-based influence propagation in social networks. In Proceedings of IEEE International Conference on Agents. Springer, 2016. 
[33] Weihua Li, Quan Bai, Chang Jiang, and Minjie Zhang. Stigmergybased influence maximization in social networks. In Proceedings of Pacific Rim International Conference on Artificial Intelligence, pages 750-762. Springer, 2016.

[34] Meng Han, Mingyuan Yan, Zhipeng Cai, and Yingshu Li. An exploration of broader influence maximization in timeliness networks with opportunistic selection. Journal of Network and Computer Applications, 63:39-49, 2016.

[35] Frédéric Derolan. Formation of social networks and diffusion of innovations. Research policy, 31(5):835-846, 2002.

[36] Shlomo Berkovsky and Jill Freyne. Personalized social network activity feeds for increased interaction and content contribution. Frontiers in Robotics and AI, 2:24, 2015.

[37] Marie \& Carlton. Which social media platforms use an algorithm and what does that mean for marketing?, March 2015. [Online; posted 5-March-2015].

[38] Matt McGee. Edgerank is dead: Facebooks news feed algorithm now has close to 100k weight factors, August 2013. [Online; posted 16-August-2013].

[39] Le Wu, Yong Ge, Qi Liu, Enhong Chen, Bai Long, and Zhenya Huang. Modeling users preferences and social links in social networking services: A joint-evolving perspective. In Thirtieth AAAI Conference on Artificial Intelligence, 2016.

[40] Fabrício Benevenuto, Tiago Rodrigues, Meeyoung Cha, and Virgílio Almeida. Characterizing user behavior in online social networks. In Proceedings of the 9th ACM SIGCOMM Conference on Internet Measurement Conference, IMC '09, pages 49-62, New York NY, USA, 2009. ACM.

[41] Xiao Fang, Paul Jen-Hwa Hu, Zhepeng Li, and Weiyu Tsai. Predicting adoption probabilities in social networks. Information Systems Research, 24(1):128-145, 2013.

[42] Wei Chen, Yajun Wang, and Siyu Yang. Efficient influence maximization in social networks. In Proceedings of the 15th ACM SIGKDD International Conference on Knowledge Discovery and Data Mining, pages 199-208, Paris, France, 2009. ACM.

[43] Julian J McAuley and Jure Leskovec. Learning to discover social circles in ego networks. In NIPS, volume 2012, pages 548-56, 2012.

[44] Bryan Klimt and Yiming Yang. Introducing the enron corpus. In CEAS, 2004

[45] Jure Leskovec, Daniel Huttenlocher, and Jon Kleinberg. Signed networks in social media. In Proceedings of the SIGCHI conference on human factors in computing systems, pages 1361-1370. ACM, 2010.

[46] Renana Peres. The impact of network characteristics on the diffusion of innovations. Physica A: Statistical Mechanics and Its Applications, 402:330-343, 2014.
Weihua Li received his M.Tech. Degree (2014) from National University of Singapore. Weihua is currently working toward the Ph.D. degree at the School of Engineering, Computer Mathematical Sciences, Auckland University of Technology, New Zealand. His research interests include influence diffusion analysis in social networks, agent-based modelling and simulation in complex systems, problem-solving using artificial intelligence techniques. He has seven publications in related fields.

Quan Bai is currently a senior lecturer at the Auckland University of Technology, New Zealand. He received his PhD (2007) and MSc (2002) from the University of Wollongong, Australia. After he had received his $\mathrm{PhD}$, Quan worked as a Postdoctoral Research Follow for the University of Wollongong, and for the Commonwealth Scientific and Industrial Research Organisation (CSIRO). Dr Quan Bai's research mainly focuses on multi-agent coordination, trust analysis and mining and agent-based modelling for complex systems.

Minjie Zhang received her B.Sc. degree in Computer Science from Fudan University, China in 1982 and her PhD degree in Computer Science from The University of New England, Australia in 1996. She is currently a Professor of Computer Science at the University of Wollongong, Australia. She is an active Researcher and has published over 200 research papers. Her research interests include multi-agent systems, agent-based modelling and simulation in complex domains, smart grid systems, and data mining and knowledge discovery in open environments.

Tung Doan Nguyen is currently pursuing the $\mathrm{PhD}$ degree at the Schoo of Engineering, Computer and Mathematical Sciences, Auckland University of Technology (AUT), New Zealand. He received the B.Eng. Degree in telecommunication from Shanghai University (2007) and MSc of the same major from Paris-Sud University (2011). His current research interests include trust management and agent-based modelling in complex systems. 